\title{
Stability Analysis of Fractal Dimension in Retinal Vasculature
}

\author{
Fan Huang ${ }^{1}$, Jiong Zhang ${ }^{1}$, Erik J. Bekkers ${ }^{1}$, Behdad \\ Dashtbozorg $^{1}$, and Bart M. ter Haar Romeny ${ }^{2,1}$ \\ 1 Department of Biomedical Engineering, Eindhoven University of Technology, \\ Eindhoven, The Netherlands \\ 2 Department of Biomedical and Information Engineering, Northeastern University, \\ Shenyang, China \\ \{F. Huang, J.Zhang1, E. J.Bekkers, B.Dasht.Bozorg, B.M.TerHaarRomeny\}@tue.nl
}

\begin{abstract}
Fractal dimension (FD) has been considered as a potential biomarker for retina-based disease detection. However, conflicting findings can be found in the reported literature regarding the association of the biomarker with diseases. This motivates us to examine the stability of the FD on different (1) vessel segmentations obtained from human observers, (2) automatic segmentation methods, (3) threshold values, and (4) region-of-interests. Our experiments show that the corresponding relative errors with respect to reference ones, computed per patient, are generally higher than the relative standard deviation of the reference values themselves (among all patients). The conclusion of this paper is that we cannot fully rely on the studied FD values, and thus do not recommend their use in quantitative clinical applications.
\end{abstract}

Keywords: Fractal dimension, Fractal analysis, Retinal Image Analysis

\section{Introduction}

The retinal fundus image is a direct acquisition of the human retina through the cornea and lens. It provides a noninvasive observation of the human vascular network at high resolution, as well as retinal landmarks like the optic disk and the fovea. Therefore, increasing attention has been drawn to retinal image analysis for detecting diseases like diabetic retinopathy, hypertension and other cardiovascular abnormalities [4].

Recent research has been focused on the study of the complexity of retinal vasculature by the analysis of fractal dimension (FD) $[1,3,4,7,13]$. FD has been considered as a potential biomarker for several diseases. However, some studies reported in $[3,7]$ found that control groups have lower FD than diabetes groups, while in $[1,4,13]$ they reported it is the other way around. This conflict motivated us to investigate the measurements more in detail.

There have been a few works in the literature studying the stability of FD. Wainwright et al. [12] studied the robustness of FD in terms of the changing of brightness, focus, contrast, image format and reported that FD is highly sensitive

X. Chen, M. K. Garvin, J. Liu, E. Trucco, Y. Xu (Eds.): OMIA 2015, Held in Conjunction with MICCAI 2015, Munich, Germany, Iowa Research Online, pp. 1-8, 2015. Available from: http://ir.uiowa.edu/omia/2015_Proceedings/2015/ 
to these factors. MacGillivray et al. [8] reported the segmentation threshold value significantly affects the FD. Mendonca et al. [9] found that the FD is highly dependent on both vessel segmentations and FD calculation methods.

In this paper, we examine the stability of three classic fractal dimension measurements that have been mostly used in previous studies. We compare the FDs calculated on binary vessel images, which were annotated by different human observers and generated by various automatic vessel segmentation methods. We also study the changing of threshold values for obtaining the vessel segmentations, and the effect of different regions of interest (ROI) for FD calculation.

The paper is organized as follows: in section 2, we introduce the classic fractal dimension methods that are widely used in clinical studies and three state-of-theart vessel segmentation methods. In section 3, we show the comparison results for different cases, and finally the conclusion is presented in section 4 .

\section{Methodology}

In this paper, we are mainly interested in three methods that are widely used in the literature: the box dimension $\left(D_{B}\right)$, information dimension $\left(D_{I}\right)$ and correlation dimension $\left(D_{C}\right)$.

\subsection{Fractal dimension measurements}

Box Dimension is the most simple and popular method for estimating the FD of fractal objects. It is the direct implementation of the Hausdorff dimension in mathematics [5], in which the dimension is defined as the real number $D_{H}$, such that the number $\mathrm{N}(r)$ of balls with radius $r$ that is needed to cover an object grows with $(1 / r)^{D_{H}}$ as $r \rightarrow 0$. In other words, $D_{H}$ is calculated via:

$$
D_{H}=\lim _{r \rightarrow 0} \frac{\log N(r)}{\log 1 / r} .
$$

In the image domain, squared boxes, instead of balls, with different sidelength $\delta$ are used to cover the whole domain, and then the number of boxes $N(\delta)$ that overlap with the structure are counted. We plot the $\log (1 / \delta)$ against $\log N(\delta)$ and the limit of Eq. (1) can be estimated by the slope of the regression line that fits to these logarithmic points.

Information Dimension was inspired from entropy theory, and is the measure of the uncertainty of a random event. It is defined as:

$$
D_{I}=\lim _{\delta \rightarrow 0} \frac{\sum_{i=1}^{N} p_{i} \log p_{i}}{\log 1 / \delta},
$$

where $N$ is the number of boxes with size $\delta$ overlapped with the object, the numerator $\sum_{i=1}^{N} p_{i} \log p_{i}$ is the first order Shannon entropy, $p_{i}=n_{i} / M$ is the probability for finding a part of the object in the $i$ th box, $M$ is the total mass of it and $n_{i}$ is the part of object in the box. The limit of Eq. (2) can also be estimated as the slope of the regression line of the logarithmic points. 
Correlation Dimension estimates the FD via the relationship between two pixels inside a region. A correlation integral is defined via the Heaviside step function for counting the pair of points in a region with size $r_{k}$, and it can be approximately expressed in terms of the probability density:

$$
C_{k}=\frac{1}{N^{2}} \sum_{\substack{i=1 \\ j=1 \\ i \neq j}}^{N} \Theta\left(r_{k}-\left\|\mathbf{x}_{i}-\mathbf{x}_{j}\right\|\right) \approx \sum_{j=1}^{N_{k}} p_{j k}^{2},
$$

where $\Theta(x)$ is the Heaviside step function, $\mathbf{x}_{i}$ is the $i$ th pixel belonging to an object, $p_{j k}=n_{j k} / M$ is the probability density of the object with mass $M$ in the $j$ th box with size $r_{k}$. The correlation dimension $D_{C}$ is defined via the power-law relationship between $C_{k}$ and $r_{k}$ as $D_{C}=\lim _{r_{k} \rightarrow 0} \frac{\log C_{k}}{\log r_{k}}$.

\subsection{Automatic vessel segmentations}

The fractal dimensions are calculated on a vessel binary map, which has pixel intensity 1 at the vessels and 0 at the background. In this work, we investigated three vessel segmentation methods which are briefly described in the following sections. The vessel probability map generated by each method is shown in Fig.1.

Frangi's vesselness is a multi-scale vessel enhancement method proposed by Frangi et al. [6], which uses the second order derivative to enhance elongated structures in the image. An important property for an elongated structure is a large change of gradient in one direction, but little gradient change in the direction perpendicular to the former. The vessels can then be enhanced by a normal probability distribution function: $\exp \left(-\frac{R_{A}^{2}}{2 \alpha}\right)\left(1-\exp \left(-\frac{S^{2}}{2 \beta^{2}}\right)\right)$, where $R_{A}=\lambda 2 / \lambda 1, S=\lambda 1+\lambda 2$, and where $\lambda_{1}$ and $\lambda_{2}$ are the eigenvalues of the $2 \mathrm{D}$ Gaussian Hessian. $R_{A}$ is an anisotropy (elongated) term, $S$ is a structure term.

Soares's segmentation is a supervised method for vessel enhancement proposed by Soares et al. [10]. It firstly extracts 5 features including the pixel intensity (the green channel) and 4 Gabor filter responses from the images. Afterward a supervised Gaussian Mixture Model (GMM) classification method is used to classify the pixels into vessel or background using the obtained features. The output is a probability map indicating the likelihood for a pixel being a vessel.

Zhang's method is based on describing the image as a function on an extended space of positions and orientations [14]. In their method, the image is lifted to the $3 \mathrm{D}$ space of positions and orientations via a wavelet-type transform. In the 3D domain, vessels are disentangled at crossings due to their difference in orientation. In the new space, Gaussian derivatives are used (exploiting a rotating coordinate system) in order to enhance blood vessels. The method results in crossing preserving enhancement of blood vessels. 


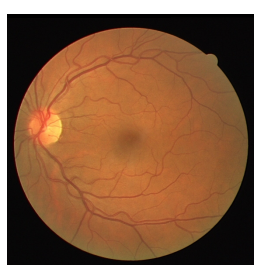

(a)

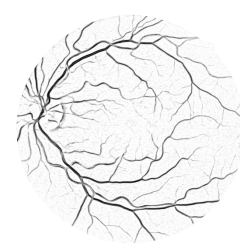

(b)

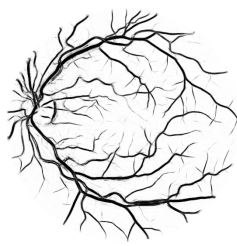

(c)

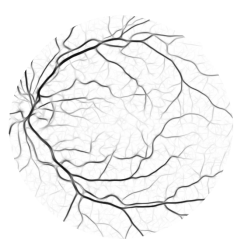

(d)

Fig. 1. (a): An original image from the DRIVE database [11]. (b)-(d): The vessel probability maps generated by the method of Frangi, Soares and Zhang, respectively.

\subsection{Region of Interest (ROI)}

In this work, the fractal dimensions were calculated in three different circular regions of interest centered at the fovea centralis with radii of 4,5 and 6 times the optic disk radius $\left(O D_{r}\right)$, respectively. The average diameter of human optic disk is $1.83 \mathrm{~mm}$ and the distance from the fovea centralis to the optic disk centralis is $4.93 \mathrm{~mm}$. Therefore, the fovea-to-OD distance is around $5 O D_{r}$. Thus the circular ROI with $4 O D_{r}$ cover the retina but excludes the optic disk, the $5 O D_{r}$ ROI covers half of the optic disk and the $6 O D_{r}$ ROI covers the full optic disk.

\section{Experimental Result}

In this section, we examine the stability of the three fractal methods in terms of the choice of manual annotations, segmentation methods, the threshold and the ROI. The 20 images, from test_1 (T1) to test_20 (T20), of the DRIVE database [11] are used in this work, which were manually annotated by two ophthalmologists. The average optic disk radius of the images is 45 pixels, so the circular ROIs centered at the fovea centralis have radii 180 (ROI 1), 225 (ROI 2) and 270 (ROI 3) pixels. The $D_{B}, D_{I}$ and $D_{C}$ are calculated on the skeletonized vessel segmentations, hence only the center lines of the vascular tree are used. The optic disk region is removed by a mask. To study the variation of FDs, we use the relative error ( $\mathrm{RE})$ with respect to the binary images annotated by observer 1 as the reference, and the Pearson Correlation coefficient ( $p$-value). The RE is obtained using $\left|\left(D_{x}-D_{r}\right)\right| / D_{r}$, where $D_{x}$ is the resulted FD and $D_{r}$ is the reference FD. In order to show the significance of these REs, the obtained values are compared with the relative standard deviations (RSD) of all subjects, which are $2.3 \%, 2.1 \%$ and $2.0 \%$ for $D_{B}, D_{I}$ and $D_{C}$, respectively.

\subsection{Comparison between two human observers}

We compared the FD values that were calculated on the ground truth images annotated by two experts within the ROI 3. Here we used the FDs of observer 1 as reference as this is also considered as ground truth in [11]. The result is shown in the 1 st row of Table 1. The main difference between the two manual annotations 


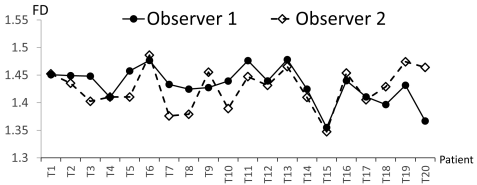

(a)

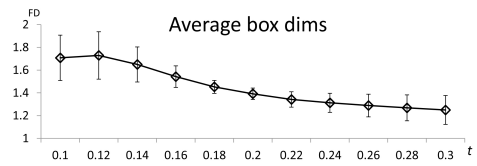

(c)

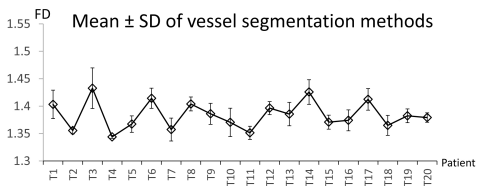

(b)

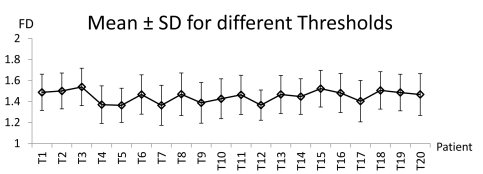

(d)

Fig. 2. (a): The $D_{B}$ of the two observers for all patients. (b): The average $D_{B}$ of three vessel segmentations. (c): The $D_{B}$ of a single image varied with the threshold. (d): The variation in $D_{B}$ of 20 patients when using different thresholds.

is in the presence of the tiny vessels. We found out that miss-detecting the tiny vessels does effect the fractal dimension, with maximal difference $7.11 \%, 6.70 \%$ and $6.23 \%$, and average difference $1.97 \%, 1.88 \%$ and $1.77 \%$ on $D_{B}, D_{I}$ and $D_{C}$, which are noticeable compared to obtained RSDs.

It means that even if the FDs are calculated on vessel maps annotated by human observers, the methods cannot produce stable values for diagnosis, which makes it less informative. In addition, Fig.2(a) plots the $D_{B}$ of 20 images of the two observers. The curves illustrate that the variations of FD for two observers in some subjects are too large which might cause a wrong discrimination among subjects for clinical applications. For example, we see the $D_{B}$ of T18 is higher than T20 for observer 1 while it is opposite for the other observer.

\subsection{Comparison among three vessel segmentations}

We investigate the variation of fractal dimension when using automatic vessel segmentation methods instead of human annotations. The methods by Frangi [11], Soares [10] and Zhang [14] were used as described previously. Each method produces a vessel probability map from the raw fundus image from which we obtain a binary map by setting an optimal threshold. For measuring the errors, we used the FDs of observer 1 as reference.

The 2 nd to 4 th rows of Table 1 show the REs made when using the binary image created by the segmentation methods instead of a human observer. Accordingly, the maximum error of the three FDs for the three segmentations are $9.4 \%, 8.9 \%$ and $7.4 \%$, respectively. The average errors are $4.28 \%, 2.94 \%$ and $3.85 \%$ which are significant compared to RSD values. These values suggest that using an automatic segmentation would make a large error in fractal calculation. In addition, the very high $p$-values imply the weak association between the automatic methods and the manual. The variation among different segmentation methods is also large according to curve shown in Fig.2(b), which shows the 
Table 1. The comparison of FD between different methods and factors.

\begin{tabular}{|c|c|c|c|c|c|c|c|c|c|}
\hline \multirow{2}{*}{ Method } & \multicolumn{3}{|c|}{ Box dimension } & \multicolumn{3}{|c|}{ Information dimension } & \multicolumn{3}{|c|}{ Correlation dimension } \\
\hline & ${ }^{*} \operatorname{Max}$ & ${ }^{\dagger} \mathrm{MRE}$ & ${ }^{\ddagger} \mathrm{PCC}$ & Max & MRE & $\mathrm{PCC}$ & Max & MRE & $\mathrm{PCC}$ \\
\hline Observer 2 & $7.1 \%$ & $2.0 \%$ & 0.0585 & $6.7 \%$ & $1.9 \%$ & 0.0851 & $6.2 \%$ & $1.8 \%$ & 0.0974 \\
\hline Frangi & $9.3 \%$ & $4.3 \%$ & 0.8035 & $9.4 \%$ & $4.3 \%$ & 0.8802 & $9.4 \%$ & $4.3 \%$ & 0.6990 \\
\hline Soares & $8.7 \%$ & $2.9 \%$ & 0.4926 & $8.7 \%$ & $3.0 \%$ & 0.7339 & $8.9 \%$ & $3.0 \%$ & 0.8657 \\
\hline Zhang & $7.4 \%$ & $3.9 \%$ & 0.4950 & $7.4 \%$ & $3.8 \%$ & 0.8506 & $7.3 \%$ & $3.8 \%$ & 0.6910 \\
\hline Threshold $(t=0.1)$ & $30.5 \%$ & $20.8 \%$ & 0.4060 & $29.4 \%$ & $20.2 \%$ & 0.6810 & $28.4 \%$ & $20.2 \%$ & 0.9733 \\
\hline Threshold $(t=0.2)$ & $10.0 \%$ & $4.8 \%$ & 0.4103 & $9.6 \%$ & $4.7 \%$ & 0.5704 & $9.1 \%$ & $4.6 \%$ & 0.8610 \\
\hline Threshold $(t=0.3)$ & $18.5 \%$ & $12.7 \%$ & 0.4410 & $16.2 \%$ & $11.7 \%$ & 0.5675 & $15.0 \%$ & $10.8 \%$ & 0.8510 \\
\hline ROI 1 & $3.8 \%$ & $2.4 \%$ & $<0.001$ & $3.6 \%$ & $1.6 \%$ & $<0.001$ & $3.5 \%$ & $1.2 \%$ & $<0.001$ \\
\hline ROI 2 & $3.5 \%$ & $1.2 \%$ & $<0.001$ & $1.0 \%$ & $0.4 \%$ & $<0.001$ & $1.0 \%$ & $0.3 \%$ & $<0.001$ \\
\hline
\end{tabular}

mean and standard deviation of $D_{B}$ among the 3 methods. This suggests that the fractal measurement is very sensitive to the choice of segmentation methods.

\subsection{Comparison of different thresholds}

We study the stability of the FD by tuning the threshold value, which is used to convert the vessel probability map to a binary map. The comparison is based on Zhang's segmentation in ROI 3. The threshold value $(t)$ ranges from 0.1 to 0.3 with step size 0.02 . The proposed optimal $t$ is 0.2 , so $t=0.1$ is an overestimation as more small structures appear (including noise) and $t=0.3$ is an underestimation because only the big structures are preserved. The FDs calculated on images of observer 1 are used as reference for error calculation.

The relative errors of changing the segmentation threshold regarding to the reference one is shown by the 5 th to 7 th row in Table 1 . Note that only the results of $t=0.1,0.2$ and 0.3 are shown in the table. From the table we see that if the threshold is set properly $(t=0.2)$, the relative error is small. While if $t$ is under- or over-estimated, the relative error dramatically increases. Moreover, Fig.2(c) shows the mean $D_{B}$ of 20 images for each threshold value, and the error bar is the average deviation compared to the ground truth. Fig.2(d) plots the mean $D_{B}$ of different threshold of each patient, and the error bar is the standard deviation of different threshold. The results suggest that improper selection of the threshold value leads to a large error for fractal dimension calculation.

\subsection{Comparison of different ROIs}

We calculated the FD in various circular regions around the fovea center of the DRIVE ground truth images annotated by observer 1. As mentioned previously, 


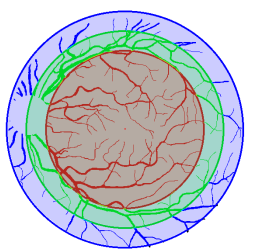

(a)

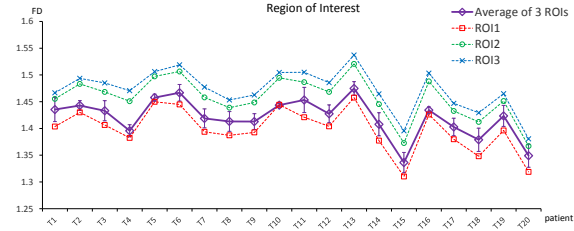

(b)

Fig. 3. (a) ROIs which are applied to the vessel map, (red, green and blue circle are the ROIs with radii 180,225 and 270 respectively). (b)The $D_{B}$ of 20 subjects varied with the change of the ROI.

the radii of ROI is 180 (ROI 1), 225 (ROI 2) and 270 (ROI 3) pixels, and ROI 3 is used as reference for the relative error calculation (see Fig.3(a)).

The relative errors of changing the ROI are shown in 8th and 9th rows in Table 1. When FDs are calculated in ROI 1, the maximum error, in terms of the three fractal, is $3.75 \%, 3.58 \%$ and $3.52 \%$, and the average error is $2.35 \%, 1.55 \%$ and $1.20 \%$, respectively. If we use ROI2, the relative errors were smaller, with an average $0.43 \%, 0.31 \%$ and $0.29 \%$. Fig. $3(\mathrm{~b})$ shows the plot of the $D_{B}$ calculated in the ROI 1 (red), ROI 2(green) and ROI 3 (blue) respectively, and also the mean and deviation of them (purple). According to Table 1 and Fig.3(b), changing the ROIs causes a variation in fractal calculation, especially the FDs of ROI 1 are significantly lower than the ROI 2 and ROI 3. But from another point of view, we see that the $p$-values are less than 0.001 , which means the FDs calculated in different ROIs are significantly associated.

\section{Discussion and Conclusion}

In previous studies, fractal dimension is considered as a potential biomarker for disease detection. We examined the reliability of three classic fractal measurements for their use in clinical studies, using four experiments.

The experimental result shows that the classic FD calculated on the binary vessel images are not reliable. The RSD of $D_{B}, D_{I}, D_{C}$ of the ground truth images are $2.3 \%, 2.1 \%$ and $2.0 \%$. The REs on measured FD values, obtained with different settings, are generally larger than the RSD among subjects. This makes the FD less informative and less reliable in clinical applications. Moreover, according to the MRE and the $p$-values in Table 1, the use of automatic vessel segmentations results in large errors, and the FD values are disassociated with the ground truth. Obtaining the FD from the segmentations of a second observer gives less error. The FD measures are least sensitive to changes in the ROI specification. When using automatic vessel segmentation methods large errors are made if the threshold is over- or underestimated.

In conclusion, our experiments suggest that the classic fractal dimensions must be calculated under very strict conditions. I.e., the vessel segmentation 
method must be very carefully chosen. An optimal threshold value for creating a binary vessel segmentation map is required. The region of interest in all images must be equally set for the FD calculation. In future work, we plan to avoid the high sensitivity for the choice of segmentation methods, by working directly on vessel probability maps (e.g. as done in $[2,7]$ ). In this case, the choice of threshold value is avoided, which was found to create the largest error.

\section{References}

1. Aliahmad, B., Kumar, D.K., Sarossy, M.G., et al.: Relationship between diabetes and grayscale fractal dimensions of retinal vasculature in the Indian population. BMC Ophthalmol 14, 152 (2014)

2. Azemin, M.Z., Kumar, D.K., Wong, T.Y., et al.: Robust methodology for fractal analysis of the retinal vasculature. IEEE Trans Med Imaging 30(2), 243-250 (2011)

3. Broe, R., Rasmussen, M.L., Frydkjaer-Olsen, U., et al.: Retinal vascular fractals predict long-term microvascular complications in type 1 diabetes mellitus: the Danish Cohort of Pediatric Diabetes 1987 (DCPD1987). Diabetologia 57(10), 2215$2221(2014)$

4. Cheung, N., Donaghue, K.C., Liew, G., et al.: Quantitative assessment of early diabetic retinopathy using fractal analysis. Diabetes Care 32(1), 106-110 (2009)

5. Falconer, K.J.: Fractal geometry : mathematical foundations and applications. J. Wiley \& sons (1990)

6. Frangi, A.F., Niessen, W.J., Vincken, K.L., et al.: Multiscale vessel enhancement filtering pp. 130-137 (1998)

7. Grauslund, J., Green, A., Kawasaki, R., et al.: Retinal vascular fractals and microvascular and macrovascular complications in type 1 diabetes. Ophthalmology 117(7), 1400-1405 (2010)

8. MacGillivray, T.J., Patton, N., et al.: A reliability study of fractal analysis of the skeletonised vascular network using the "box-counting" technique. Conf Proc IEEE Eng Med Biol Soc 1, 4445-4448 (2006)

9. Mendonca, M.B., Amorim, G., et al.: Fractal analysis of retinal vascular tree: segmentation and estimation methods. Arq Bras Oftalmol 70(3), 413-422 (2007)

10. Soares, J.V., Leandro, J.J., et al.: Retinal vessel segmentation using the 2-D Gabor wavelet and supervised classification. IEEE Trans Med Imaging 25(9), 1214-1222 (2006)

11. Staal, J., Abramoff, M., Niemeijer, M., et al.: Ridge based vessel segmentation in color images of the retina. IEEE Transactions on Medical Imaging 23(4), 501-509 (2004)

12. Wainwright, A., Liew, G., Burlutsky, G., et al.: Effect of image quality, color, and format on the measurement of retinal vascular fractal dimension. Invest. Ophthalmol. Vis. Sci. 51(11), 5525-5529 (2010)

13. Yau, J.W., Kawasaki, R., et al.: Retinal fractal dimension is increased in persons with diabetes but not impaired glucose metabolism: the Australian Diabetes, Obesity and Lifestyle (AusDiab) study. Diabetologia 53(9), 2042-2045 (2010)

14. Zhang, J., Bekkers, E., Abbasi, S., et al.: Robust and fast vessel segmentation via gaussian derivatives in orientation scores. Accepted by ICIAP 2015 pp. 1-11 (2015) 